\title{
Effect of Loop Structure of Bovine Lactoferricin on Escherichia Coli
}

\author{
Rui Xu*, Xi-yan Zhao, Shuo Guo, Qi Yang \\ College of Food Science and Technology, Hebei Normal University of Science and Technology, \\ Qinhuangdao, 066004, China \\ *E-mail: robust100@163.com
}

\begin{abstract}
As a glycoprotein, lactoferrin has many biological actions, among which the antibacterial activity is one of the most important effects studied. Bovine lactoferricin (Lfcin B) is a cationic peptide that possesses potent in vitro and in vivo antibacterial activity, and might affect the expression of DNA Adenine Methyltransferase (DAM). In the present study, the artificially synthesized Lfcin B and Lfcin B derived peptide (Cys 19 residue in Lfcin B was replaced by Ala) were added in Escherichia coli, and we determined the messenger ribonucleic acid and protein expression of DAM in Escherichia coli after incubated with Lfcin B by real-time quantitative reverse transcription PCR and Western blot analysis. The results of real-time quantitative reverse transcription PCR showed that DAM expression was reduced after treatment with Lfcin B. The results of western blot analysis showed that the expression of DAM protein was down-modulated by Lfcin B.
\end{abstract}

Keywords: Lactoferrin, DNA Adenine Methyltransferase, western blot, Escherichia coli

\section{Introduction}

Lfcin B is a 25-residue peptide fragment discharged from the N-terminal domain of bovine lactoferrin (residues $17 \sim 41$ ) upon pepsin digestion, bovine lactoferrin $(\mathrm{LF})$ is an $80 \mathrm{kDa}$ iron-binding glycoprotein that is found in the secretory granules of neutrophils and in biological fluids, including saliva and milk. Lfcin B is a cationic antimicrobial peptide with cytotoxic activity against microorganisms[1]. It consists of 25 amino acid residues (17 to 41 proximal to the NH2 terminus of bovine lactoferrin) and is produced by acid-pepsin hydrolysis of bovine lactoferrin. Lfcin B is an antimicrobial peptide that displays no iron-binding capacity[2]. Studies have demonstrated that the mammalian LF and Lfcin B in addition to activities reported against fungi and protozoa exert antibacterial activities[3,4].

DNA methylation plays a significant role in the tissue- and stage-specific modulation of genes, although the molecular basis of the relationship between Lfcin B and methylation of Escherichia coli is not yet clear, one possibility is that the cationic, amphipathic of Lfcin B could affect the expression of DAM[5].

Lfcin B features a loop region due to a disulfide bridge between Cys 19 and Cys 36. Jenssen et al. suggested that the loop structure through a disulfide bond was important for antiviral activity[6]. But currently, no interrelated study was reported about influence of the loop structure of Lfcin B on inducing apoptosis in Escherichia coli. A link between Lfcin B and DAM expression in Escherichia coli has not been established. We investigated the effects of Lfcin B on the down-modulation of DAM expression in Escherichia coli.

\section{$2 \quad$ Materials and Methods}

\section{Materials}

Lfcin B (amino acid sequence: FKCRRWQWRMKKLGAPSITCVRRAF) derivatives were synthesized in linear form by Sangon (Sangon, Shanghai, China). E. coli strain was obtained from the China Center of Industrial Culture Collection (CICC, Beijing, China). After a 50- $\mu$ l culture aliquot of the microorganism was added to tryptic soy yeast extract broth $(9 \mathrm{~mL})$, it was incubated at $37^{\circ} \mathrm{C}$ for $20 \mathrm{~h}$. Then, it was preserved at $-30^{\circ} \mathrm{C}$ after adding $15 \%$ glycerol for use as stock cultures. 


\section{Antibacterial Activity Assays}

The antibacterial activities were detected using the approach employed by Balouiri et al.[7]. Samples were divided into three groups, group 1: the sample was cultured for $12 \mathrm{~h}$ without Lfcin B; group 2: the sample was cultured for $12 \mathrm{~h}$ with Lfcin B $(50 \mu \mathrm{g} / \mathrm{ml})$; group 3: the sample was cultured for $12 \mathrm{~h}$ with Lfcin B-derived peptide $(50 \mu \mathrm{g} / \mathrm{ml})$.

\section{Real-time Quantitative Reverse Transcription PCR}

Cultured cells were washed twice with phosphate buffered saline (PBS) and harvested. Total RNA from cells was isolated using the MagMAX ${ }^{\text {TM}}$-96 Total RNA Isolation Kit (ABI-Ambion, TX, USA) according to the manufacturer's instructions. Real-time quantitative PCR was carried out in 96-well polypropylene microplates on an ABI Prism 7500 (Applied Biosystems, CA, USA) using SYBR Green Real-time PCR Master Mix (TOYOBO, Tokyo, Japan) according to the manufacturer's instructions[8]. The data analyses were performed according to the method introduced previously[9].

\section{Western Blot Analyses}

Cells were lysed in protein lysis buffer containing $7 \mathrm{~mol} / \mathrm{L}$ of urea, $2 \mathrm{~mol} / \mathrm{L}$ of thiourea, $4 \%$ CHAPS, 65 $\mathrm{mmol} / \mathrm{L}$ of DTT, $0.2 \%$ (pH 3-10) Bio-Lyte (Bio-Rad, Hercules, CA), and complete protease cocktail inhibitors (Roche, Laval, Canada). Lysates were ultrasonicated and cleared by centrifugation at $13,000 \mathrm{~g}$ at $4^{\circ} \mathrm{C}$ for $10 \mathrm{~min}$ and the supernatant was collected. Proteins were quantified by using the Bradford method. Samples were boiled in SDS sample buffer and total protein $(20 \mu \mathrm{g})$ was loaded into each well of an $8 \%$ SDS-polyacrylamide gel for separation by electrophoresis. Protein bands were transferred onto nitrocellulose membranes. The resulting blots were blocked overnight with PBS-Tween 20 (0.25 mol/L Tris, $150 \mathrm{mmol} / \mathrm{L} \mathrm{NaCl}, 0.2 \%$ Tween 20 in PBS) containing 3\% powdered skim milk and then probed overnight with the desired primary antibody at a 1:500 dilution. Blots were then washed with PBS-Tween 20 and probed for 1 hour with HRP-conjugated second antibody (1:10, 000) as appropriate. Following additional washes with PBS-Tween 20, the protein bands were visualized using an enhanced chemiluminescence detection system (GE Healthcare, Piscataway, NJ, USA).

\section{Statistical Analysis}

The experiments were carried out three times using freshly prepared samples. Microsoft Excel 2010 was applied to calculate mean and standard deviations based on all the data obtained in the experiments. Statistical evaluation was conducted for the data by ANOVA on the basis of Tukey test using the SPSS 19.0 package.

\section{$3 \quad$ Results}

\section{Effect of Lfcin B Loop Structure on Antibacterial Activity}

E. coli cells were incubated in the presence or absence of Lfcin B at a concentration of $50 \mu \mathrm{g} / \mathrm{mL} \mathrm{for} 12 \mathrm{~h}$. Lfcin B was shown to have significant inhibition effects on E. coli $(\mathrm{p}<0.05)$ (Figure 1$)$. The highest decrease (30 log cycles) relative to the control was found with Lfcin B after incubation. Figure 1 shows that Lfcin B significantly inhibited Escherichia coli, but LfcinB-derived peptide did not inhibit Escherichia coli.

\section{Lfcin B Down-modulates the Level of DAM mRNA}

E. coli cells were incubated in the presence or absence of Lfcin B at a concentration of $50 \mu \mathrm{gg} / \mathrm{mL}$ for $12 \mathrm{~h}$. After incubation, total RNA was isolated and treated with DNase I, quantified, and reverse-transcribed into cDNA. The DAM transcript levels were measured by real-time quantitative reverse transcription PCR analysis of cDNA. The results were standardized to $\beta$-actin levels. Treatment with Lfcin B significantly caused a 2-fold reduction in DAM mRNA level (Figure 2), however, treatment with LfcinB-derived peptide did not cause a reduction in DAM mRNA level. 


\section{Lfcin B Down-modulates DAM Protein Levels}

Proteins were separated using $8 \%$ SDS-PAGE. After transferring and blocking, they were reacted with primary antibodies at $4^{\circ} \mathrm{C}$ overnight. Target proteins were subsequently detected using HRP-conjugated IgG with ECL plus and Microtek Scanner (Microtek, Shanghai, China). Changes in DAM protein content were detected by Western blotting. Lfcin B treatment progressively reduced DAM protein levels $40 \%$ (Figure 3).

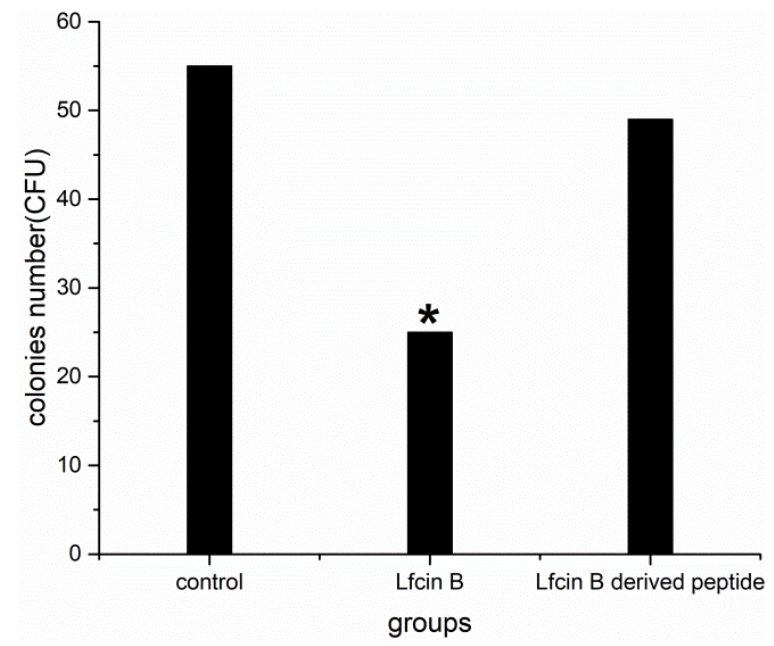

Figure 1. The different effects of Lfcin B and Lfcin B-derived on Escherichia coli. Values are the mean \pm standard deviation from three replicates of three independent experiments. An asterisk indicates significant differences $(p<0.05)$ with respect to the control.

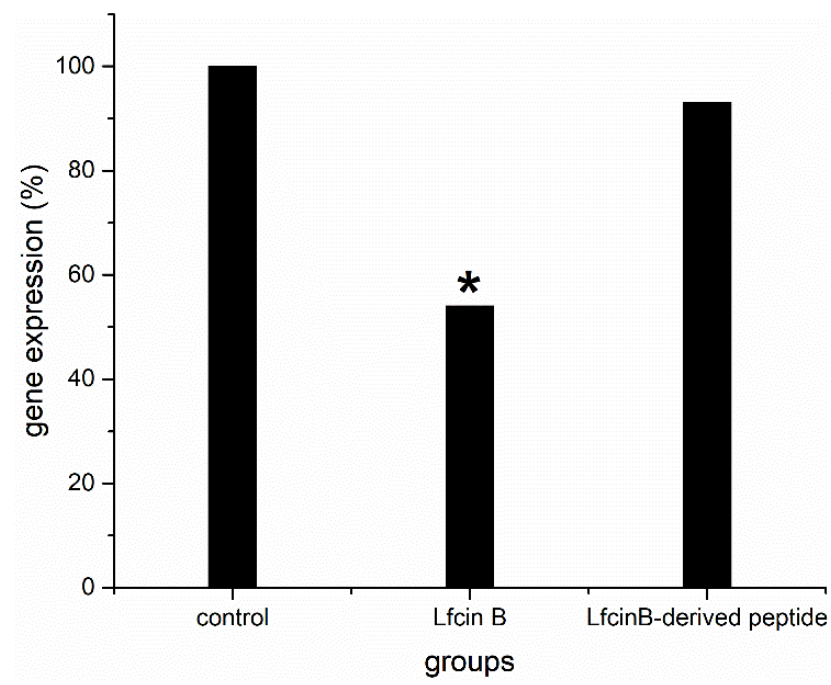

Figure 2. Down-modulation of DAM mRNA expression by Lfcin B. Real-time quantitative reverse transcription PCR results were expressed as percentage of their respective controls. Three samples were measured for each group. An asterisk indicates significant differences $(\mathrm{p}<0.05)$ with respect to the control. 


\section{LfcinB-derived Lfcin B peptide}

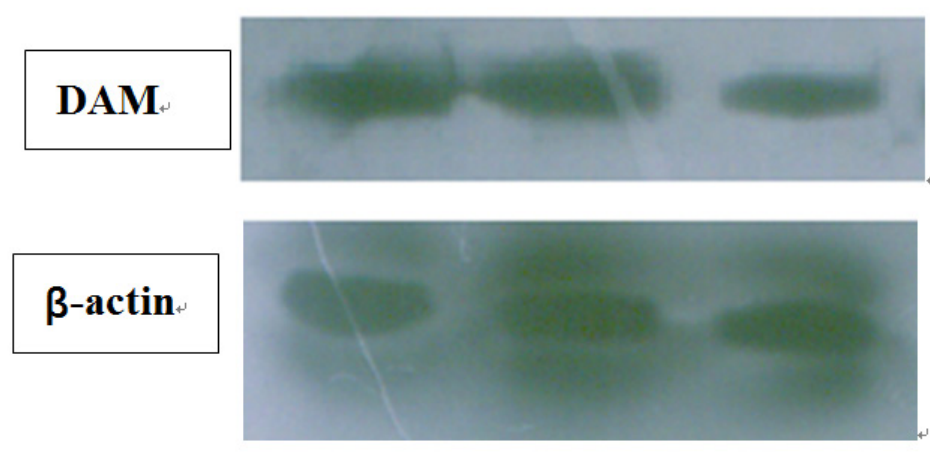

Figure 3. Down-modulation of DAM protein expression by Lfcin B

\section{Discussion}

In recent years, great attentions have been paid on bioactive components obtained from food, particularly those showing antimicrobial activities. As a matter of fact, Lfcin B has been widely used as a supplement of functional and infant products attributed to its various functions, including immunomodulatory and antibacterial activities. Although Lfcin B is normally added to products not as a whole protein, the peptides discharged from it after enzymatic hydrolysis have been investigated extensively during the last two decades because of its strengthened antibacterial activities[10]. Both LF and Lfcin B, with their relative high net positive charge, may interact with negatively charged GAG chains expressed on the target cells[11], and thereby display antiviral activities. Apoptosis induction was triggered by a sequence of events comprising Lfcin B-mediated permeabilization of the cell membrane, Lfcin B aggregation on the sides of mitochondria, and subsequent depolarization of the mitochondrial membrane. This led to the release of cytochrome $\mathrm{C}$ and initiation of the intrinsic pathway of apoptosis[12]. They also discovered that modulation of ceramide metabolism was involved in the induction of apoptosis induced by Lfcin B[13].

However, effect of bovine lactoferricin on DAM levels in E. coli cells has been completely understood. In this study, Lfcin B decreased the level of DAM mRNA in E. coli cells. Western blots analysis revealed a reduction of DAM protein expression in cells. Our results indicated that the activity of Lfcin B reduced the stability of DAM mRNA, and that this activity could be reduced by protein synthesis. These results, in conjunction with those of the current study, suggest that down-modulation of DAM gene expression by Lfcin B. These data support our hypothesis that Lfcin B might affect the expression of DAM and the stability of DAM mRNA in E. coli cells. The cytotoxic activity of Lfcin B in E. coli cell lines suggests that it could be potentially useful for the treatment of certain bacterial microbes.

Lfcin B possessed potent in vitro antibacterial activity. In vitro exposure to Lfcin B caused apoptosis in several microbes. Lfcin B formed a characteristic loop structure resulting from a disulfide bridge between Cys 19 and Cys 36, the deficiency of this loop structure could lose or weaken the antivirus and antibiotic activity[6], the present investigation indicated that existence of Cys 19 in Lfcin B had important influence on inducing apoptosis in Escherichia coli, LfcinB-derived peptide could neither induce apoptosis in Escherichia coli, nor improve the DAM level, deficiency of the loop structure could cause Lfcin B losing the biological activity of antibacterial activity.

\section{Conclusions}

Lfcin B produces antimicrobial peptides activities against E. coli with very low concentrations. These results show that it is promising to use Lfcin B as a functional ingredient or as a food preservative in the future. However, further research is expected to be carried out to clarify the activities of Lfcin B in a complex food system. This enables people to obtain protein fractions with strong antimicrobial effects and add them in food products. Therefore, Lfcin B is supposed to become a promising approach to relieve 
colonization pressure on farms and to prevent E. coli pollution of food and, hence to lessen human illness associated with E. coli.

\section{Reference}

1. F. F. Han, Y. H. Gao, C. Luan. "Comparing bacterial membrane interactions and antimicrobial activity of porcine lactoferricin-derived peptides," Journal of Dairy Science, Vol. 96, no. 6, pp. 3471-3487, 2013.

2. W. Bellamy, M. Takase, H. Wakabayashi, K. Kawase and M. Tomita. "Antibacterial spectrum of lactoferricin B, a potent bactericidal peptide derived from the N-terminal region of bovine lactoferrin," Journal of Applied Bacteriology, Vol. 73, no. 1, pp. 472-479, 1992.

3. Y. Omata, M. Satake, R. Maeda, A. Saito, K. Shimazaki, K. Yamauchi, Y. Uzuka, S. Tanabe, T. Sarashina, T. Mikami. "Reduction of the infectivity of Toxoplasma gondii and Eimeria stiedai sporozoites by treatment with bovine lactoferricin," Journal of Veterinary Medical Science, Vol. 63, no. 2, pp. 187-190, 2001.

4. J. H. Andersen, H. Jenssen, T. J. Gutteberg. "Lactoferrin and lactoferricin inhibit herpes simplex 1 and 2 infection and exhibit synergy when combined with acyclovir," Antiviral Research, Vol. 58, no. 4, pp. 209-215, 2003.

5. A. Shestakov, H. Jenssen, I. Nordström. "Lactoferricin but not lactoferrin inhibit herpes simplex virus type 2 infection in mice," Antiviral Research, Vol. 93, no. 3, pp. 340-345, 2012.

6. H. Jenssen, J. H. Andersen, L. Uhlin-Hansen, T. J. Gutteberg, Ø. Rekdal. "Anti-HSV activity of lactoferricin analogues is only partly related to their affinity for heparansulfate," Antiviral Research, Vol. 61, pp. 101-109, 2004.

7. M. Balouiri, M. Sadiki, S. K. Ibnsouda. "Methods for in vitro evaluating antimicrobial activity," Journal of Pharmaceutical Analysis, Vol. 6, no. 2, pp. 71-79, 2016.

8. S. Poojariab, O. J. Alabi. "Green-based real-time quantitative reverse-transcription PCR for detection and discrimination of grapevine viruses," Journal of Virological Methods, Vol. 235, no. 2, pp. 112-118, 2016.

9. J. L. Kenneth and D. S. Thomas. "Analysis of Relative Gene Expression Data Using Real-Time Quantitative PCR and the $2^{-\Delta \Delta \mathrm{C}_{\mathrm{T}}}$ Method," Methods, Vol. 25, no. 1, pp. 402-408, 2001.

10. N. Bruni, M. T. Capucchio, E. Biasibetti. "Antimicrobial Activity of Lactoferrin-Related Peptides and Applications in Human and Veterinary Medicine," Molecules, Vol. 21, no. 6, pp. 752-755, 2016.

11. E. Yazidi-Belkoura, D. Legrand, J. Nuijens. "The binding of lactoferrin to glycosaminoglycans on enterocyte-like HT29-18-C1 cells is mediated through basic residues located in the N-terminus," Biochimica et Biophysica Acta, Vol. 1568, no. 3, pp. 197-204, 2001.

12. J. S. Mader, A. Richardson, J. Salsman, D. Top, R. Antueno, R. Duncan and D. W. Hoskin. "Bovine lactoferricin causes apoptosis in Jurkat T-leukemia cells by sequential permeabilization of the cell membrane and targeting of mitochondria," Experimental Cell Research, Vol. 313, no. 1, pp. 2634-2650, 2007.

13. S. Furlong, N. Ridgway, D. W. Hoskin. "Modulation of ceramide metabolism in T-leukemia cell lines potentiates apoptosis induced by the cationic antimicrobial peptide bovine lactoferricin," International Journal of Oncology, Vol. 32, no. 1, pp. 537-544, 2008. 